\title{
Mössbauer Studies of Surface Modified Magnetite Particles
}

\author{
U. Klekotka ${ }^{a}$, E. WińskA ${ }^{a}$, D. SAtuŁA ${ }^{b}$ And B. Kalska-Szostko ${ }^{a *}$ \\ ${ }^{a}$ Institute of Chemistry, University of Bialystok, K. Ciołkowskiego 1K, 15-245 Białystok, Poland \\ ${ }^{b}$ Faculty of Physics, University of Bialystok, K. Ciołkowskiego 1L, 15-245, Białystok, Poland
}

\begin{abstract}
The Mössbauer spectroscopy was used for the characterization of room temperature magnetic properties of magnetite/maghemite nanoparticles, in respect of surfactant. The studied nanoparticles were obtained using various types of surface stabilizers in order to see if the application of different surfaces substances has an influence on the magnetic response of nanoparticles core. Nanostructures were fabricated by co-precipitation of iron(II) and (III) chlorides in ammonia solution. Five types of organic acids were used for the stabilization of nanoparticles. Obtained nanoparticles were structurally characterized by transmission electron microscopy and X-ray diffraction. Room temperature magnetic behavior was analyzed on the base of conventional and in field (1.3 T) Mössbauer spectroscopy.
\end{abstract}

DOI: 10.12693/APhysPolA.134.1003

PACS/topics: $61.10 . \mathrm{Nz}, 75.47 . \mathrm{Lx}, 75.50 .-\mathrm{y}, 76.80 .+\mathrm{y}, 75.50 . \mathrm{Tt}, 82.70 . \mathrm{Uv}$

\section{Introduction}

Magnetite nanoparticles are a very popular type of nanomaterials, which can be obtained by various physical and chemical techniques [1-4]. Chemical synthesis of magnetite nanoparticles requires an application of proper surface stabilizers [5]. There are many different substances used as protectors from agglomeration or oxidation. The few most common are: in water - tetrabutylammonium hydroxide [6], citric acid [7], and in organic medium - oleic acid [8], oleylamine [9]. However, when choosing a proper surface stabilizer, one should remember that they could significantly influence the magnetic response of the magnetic core and also develop additional functionality. Therefore, studies of surfactant impact on magnetic properties are especially important when the application is forecasted because each system must be characterized in detail [10].

Magnetite or ferrite nanoparticles are the most common kind of nanomaterials studied in the literature $[8,11,12]$. Nevertheless, the published data is very scattered and the conclusions are only valid for particular cases. In this paper, scientists are focused on materials modification only at outermost layer, which in many cases is treated marginally, but in reality, can play an important role. Surface oxidation can trap magnetic moment orientation due to different dipoledipole interaction as oxide characteristics [13]. It was also observed that particles fabricated into two different synthetic procedures do not exhibit the same properties, especially in terms of resistance to oxidation [14]. It is a very important source of possible usability of the magnetic core.

*corresponding author; e-mail: kalska@uwb.edu.pl

\section{Experimental}

\subsection{Material and apparatus}

To obtain nanoparticles with changed synthesis routines, the following chemicals were purchased from Aldrich: tetramethylammonium hydroxide (TMAOH), phthalic anhydride, succinic anhydride, ethylenediaminetetraacetic acid. Iron(III) and (II) chlorides, ammonia solution, ascorbic acid, and acetone were obtained from POCH. Cleaning and separation of nanoparticles were performed with use of deoxygenated acetone, sonication bath, and a permanent magnet.

Synthesized nanoparticles were characterized by: X-ray diffraction (XRD) by Agilent Technologies SuperNova diffractometer with a Mo microfocused source $\left(K_{\alpha 2}=0.713067 \AA\right)$, transmission electron microscopy (TEM) FEI Tecnai G2 X-TWIN 200kV microscope, and infrared spectroscopy (IR) Nicolet 6700 spectrometer working in reflecting mode. The Mössbauer spectra were obtained with the use of the standard spectrometer working in constant acceleration mode with a $\mathrm{Co} \underline{\mathrm{Rh}}$ radioactive source. Metallic iron foil $(\alpha-\mathrm{Fe})$ was used as reference material for isomer shift estimation. All samples were measured in the transmission mode. Magnetic field $(1.3 \mathrm{~T})$ was obtained by a permanent magnet.

\subsection{Preparation of magnetite nanoparticles}

Synthetic procedure of nanoparticles is conducted in two separate 3-neck flasks. In the first one, $40 \mathrm{ml}$ of distilled water was deoxygenated for $30 \mathrm{~min}$, and in the second one, $4 \mathrm{ml}$ of distilled water and $1 \mathrm{ml}$ of $38 \% \mathrm{HCl}$ was also deoxygenated with argon. In the next step, to the first glass, $2.7 \mathrm{~g}$ of $\mathrm{FeCl}_{3} \cdot 6 \mathrm{H}_{2} \mathrm{O}$ was added, and to the second one $1 \mathrm{~g}$ of $\mathrm{FeCl}_{2} \cdot 4 \mathrm{H}_{2} \mathrm{O}$, respectively. Both constituents were then heated up to $50^{\circ} \mathrm{C}$. When it reached the same temperature, the solution from the second flask was slowly added to the first one. Then, $200 \mathrm{ml}$ of $1 \mathrm{M}$ $\mathrm{NaOH}$ solution was heated up to $100^{\circ} \mathrm{C}$ and was added. The whole mixture was boiled for 30 min until it reached 
$95^{\circ} \mathrm{C}$. Then, it was cooled down to room temperature and the precipitate was separated from the solution and washed with deoxygenated distilled water. In the next step, $0.01 \mathrm{M}$ solution of respective surfactant was used (citric acid, EDTA, ascorbic acid, phthalic anhydride, or succinic anhydride). For details and samples abbreviations see Table I. Then the solution TMAOH was added until the $\mathrm{pH}=7$ level was reached. To obtain nanoparticles in powder form, the precipitate was washed with acetone 3 times and dried on a vacuum evaporator.

Summary of types of used surfac-

TABLE I tants on prepared nanoparticles

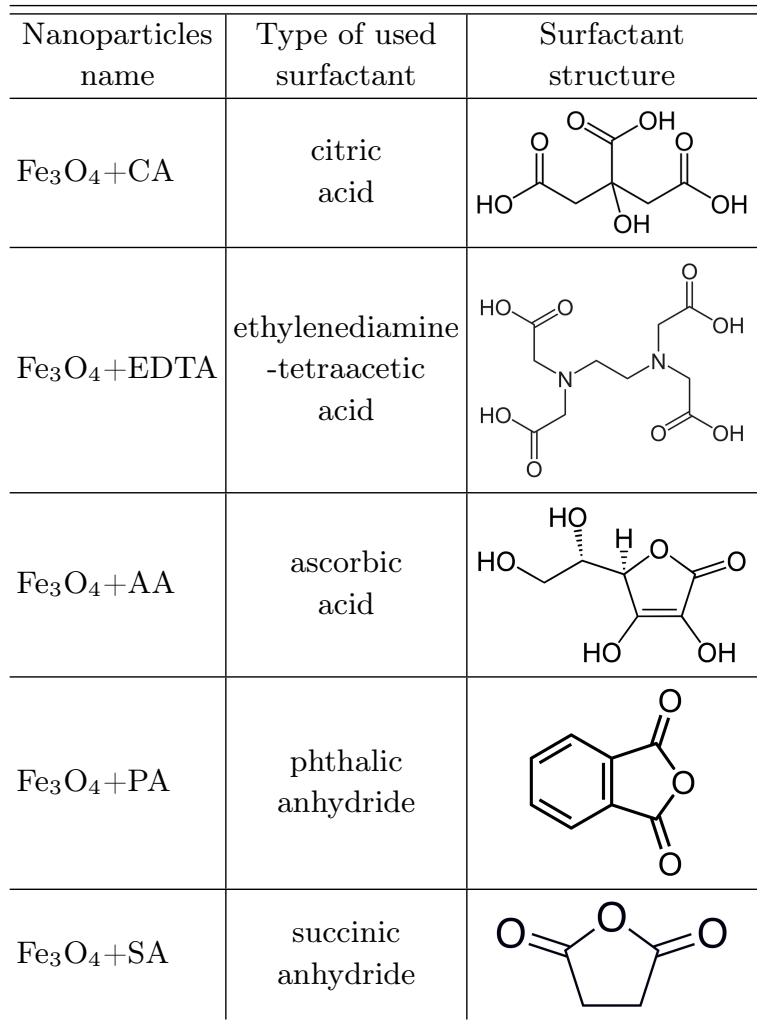

\section{Results and discussion}

\subsection{Transmission electron microscopy}

As-prepared nanoparticles were diluted in a small amount of ethanol and dropped on $\mathrm{Cu}$ grid. Images of all types of tested nanoparticles are depicted below in Fig. 1. In Table II, the calculated values of average nanoparticles size from TEM images are collected.

The presented images show that in every modified synthetic procedure case, nanoparticles were successfully obtained. However, usage of surfactants causes different growth (in respect of size and shape) of the nanoparticles and its separation. Nanoparticles in which succinic anhydride was used grow much bigger than in all other cases $(14 \pm 2 \mathrm{~nm})$ and are rather squares instead of being round. As seen in Table II, the smallest nanoparticles were obtained in the case of ascorbic acid as a surfactant $(6 \pm 2 \mathrm{~nm})$, and the most even in size and shape when EDTA was used.

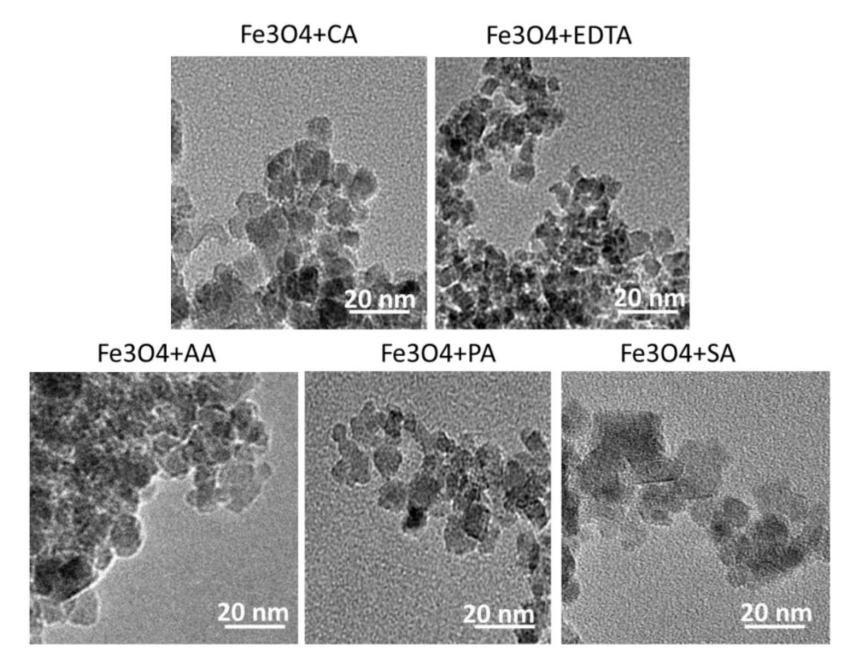

Fig. 1. TEM images of nanoparticles with various surfactants.

TABLE II

Nanoparticles size estimated from TEM images and calculated from the X-ray diffractogram together with strain, and lattice cells parameter of magnetite phase.

\begin{tabular}{l|c|c|c|c}
\hline \hline $\begin{array}{c}\text { Nanoparticles } \\
\text { name }\end{array}$ & $\begin{array}{c}\text { Size TEM } \\
{[\mathrm{nm}] \pm 2}\end{array}$ & $\begin{array}{c}\text { Size XRD } \\
{[\mathrm{nm}] \pm 2}\end{array}$ & $\begin{array}{c}\text { Strain } \\
{\left[10^{-3}\right]} \\
\pm 0.5\end{array}$ & $\begin{array}{c}\text { Lattice } \\
\text { constant } \\
{[\AA] \pm 0.02}\end{array}$ \\
\hline $\mathrm{Fe}_{3} \mathrm{O}_{4}+\mathrm{CA}$ & 10 & 7 & 3.1 & 8.34 \\
$\mathrm{Fe}_{3} \mathrm{O}_{4}+\mathrm{EDTA}$ & 8 & 7 & 1.4 & 8.37 \\
$\mathrm{Fe}_{3} \mathrm{O}_{4}+\mathrm{AA}$ & 6 & 7 & 2.6 & 8.36 \\
$\mathrm{Fe}_{3} \mathrm{O}_{4}+\mathrm{PA}$ & 9 & 7 & 0.5 & 8.34 \\
$\mathrm{Fe}_{3} \mathrm{O}_{4}+\mathrm{SA}$ & 14 & 7 & 2.9 & 8.34
\end{tabular}

\subsection{X-ray diffraction}

To observe changes in the crystalline structure of characterized nanoparticles, X-ray diffraction was applied. Obtained diffractograms for the series are depicted in Fig. 2.

Presented in Fig. 2 the set of diffraction patterns shows that regardless of surfactant in every case the same set of peaks can be observed. In addition, the line positions at $2 \theta$ angles were not changed. It proves that the inverse spinel structure of magnetite is preserved for each used surfactant. There is no evidence of oxidation to hematite during the fabrication process. However, maghemite cannot be excluded. Observed reflexes were recognized and assigned with magnetite/maghemite $h k l$ indexes (111), (220), (311), (400), (422), (511), (440), (620), respectively [15]. Calculated lattice constant varies in range $(8.34-8.37 \pm 0.02) \AA$, which is very close to the literature of bulk magnetite $8.39 \AA$ [16] or maghemite $8.33 \AA$ [17].

Obtained X-ray data served as a base for determination of the average grain size and crystallites strain. Quantitative analysis was performed using the Williamson-Hall Eq. (1) $[18,19]$. Calculated crystalline sizes are summarized in Table II, together with lattice constants and strain values: 


$$
\beta \cos \theta=\frac{0.9 \lambda}{D}+4 \varepsilon \sin \theta
$$

where $D$ - grain size $[\AA], \lambda$ - wavelength (for Mo source is $0.7136 \AA$ ), $\beta$ - full width at half maximum intensity of the peak [rad], $\varepsilon-$ strain, and $\theta-$ diffraction angle [rad].

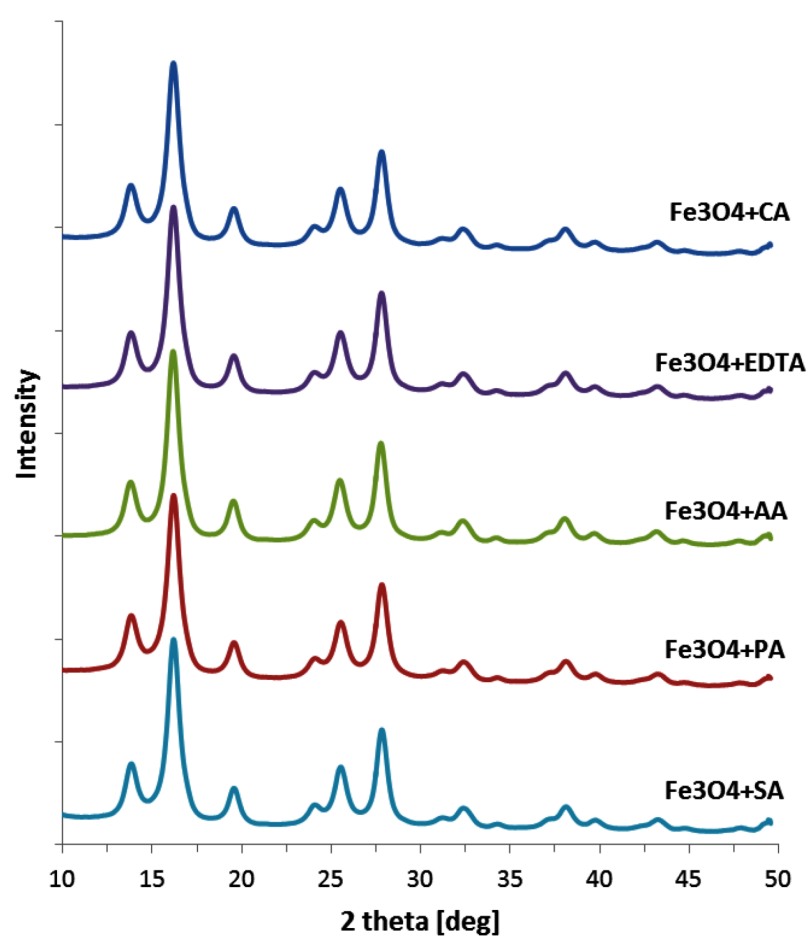

Fig. 2. X-ray patterns of nanoparticles crystal structure.

The fitting of Eq. (1) to measurement data gave the average crystallite size almost the same for each system. It is not consistent with the TEM data but taking into account the number of different contributions, which can influence the lines broadening, does not make findings invalid. The largest particles (according to TEM) in XRD simulation have the highest values of strain which was obtained and suggest that other contributions to the calculated size have had a significant impact [19]. The most important is, however, that requested cores were obtained.

\subsection{Mössbauer spectroscopy}

Nanoparticles magnetic response as a function of used surfactants was studied on the base of room temperature (RT) Mössbauer spectroscopy. The measurements were carried out with and without an external magnetic field of the value $1.3 \mathrm{~T}$ parallel to the beam direction. In Fig. 3, two sets of the spectra are depicted. In part (a) data obtained without external magnetic field are collected, while in part (b) spectra of the same samples measured in a magnetic field are presented.

RT Mössbauer spectra collected without external magnetic field show superparamagnetic doublet in the central

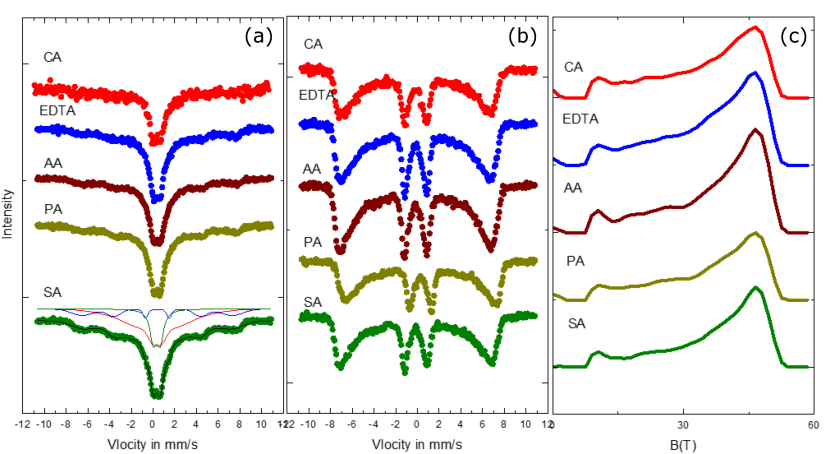

Fig. 3. The Mössbauer spectra of tested nanoparticles (a) without the external magnetic field, (b) with external magnetic field presence, (c) hyperfine magnetic field distribution curves. The solid lines represent the best fit of representative spectrum SA, on the bottom of the part (a) individual components are presented.

part of spectra and low intensive broad sextet as a background (Fig. 3a). The spectra were fitted using one doublet and two broad sextets. These components are associated with the distribution of relaxation time of the magnetic moments of iron atoms and do not correspond to the two Fe positions in the spinel structure. In addition, the measurements in the external magnetic field show that there are no separate components which come from the $\mathrm{Fe}[\mathrm{A}]$ and $\mathrm{Fe}[\mathrm{B}]$ sites of magnetite. It means that in our systems we are dealing with a mixture of maghemite and magnetite. Because the hyperfine parameters of maghemite do not differ significantly and measured spectra are broad - the isomer shift values were assumed to be the same for all components and quadrupole splitting equals $0 \mathrm{~mm} / \mathrm{s}$ for magnetic components. The analysis of the measured spectra shows that isomer shift does not change for all samples and equals $(0.35 \pm 0.03) \mathrm{mm} / \mathrm{s}$. The quadrupole splitting of the observed doublet is equal to $(0.61 \pm 0.05) \mathrm{mm} / \mathrm{s}$. Moreover, the least intensive magnetic contribution is observed for CA while SA has the highest value of sextet intensity. There are no visible correlations between the size of nanoparticles and the intensities of the magnetic part in the measured spectra which indicates the influence of the kind of surfactant on the magnetic behavior of the nanoparticles. The intensities of the magnetic part in the measured spectra are presented in Table III. Such observations can suggest the presence of dipole-dipole interaction between nanoparticles, which is weaker when smaller/shorter surfactants are used for surface stabilization. SA has the smallest volume and therefore a linear size, which can cause closer attraction between magnetic cores which has a direct impact on the collective flipping or trapping of magnetic moments.

The Mössbauer spectra measured in an external magnetic field $(1.3 \mathrm{~T})$ parallel to beam direction are presented in Fig. 3b. The superparamagnetic doublet visible in the Mössbauer spectra measured without external magnetic field disappears. It means that the magnetic interaction between iron magnetic moments and external magnetic 
TABLE III

Intensities of the magnetic part of spectra $I_{\text {mag }}$ for RT Mössbauer measurements without applied magnetic field and mean values of hyperfine field $B_{\text {field }}$ obtained from in field measurements.

\begin{tabular}{l|c|c}
\hline \hline Nanoparticls name & $I_{\text {mag }}[\%] \pm 2$ & $B_{\text {field }}[\mathrm{T}] \pm 1.2$ \\
\hline $\mathrm{Fe}_{3} \mathrm{O}_{4}+\mathrm{CA}$ & 53 & 35.2 \\
$\mathrm{Fe}_{3} \mathrm{O}_{4}+\mathrm{EDTA}$ & 60 & 35.8 \\
$\mathrm{Fe}_{3} \mathrm{O}_{4}+\mathrm{AA}$ & 56 & 36.9 \\
$\mathrm{Fe}_{3} \mathrm{O}_{4}+\mathrm{PA}$ & 58 & 36.0 \\
$\mathrm{Fe}_{3} \mathrm{O}_{4}+\mathrm{SA}$ & 70 & 37.5
\end{tabular}

field 1.3 $\mathrm{T}$ is large enough to suppress superparamagnetic fluctuation in all studied samples. The measured spectra are broad with low intensity of 2 nd and 5 th lines so the hyperfine magnetic field distributions (h.m.f.) were determined using the histogram method with smoothing. The isomer shift was assumed to be the same for whole distribution and quadrupole splitting was taken as $0 \mathrm{~mm} / \mathrm{s}$. The calculated h.m.f. distribution are presented in Fig. 3c. As one can see the shapes of h.m.f. distributions are similar for all samples with the maximum of $45 \mathrm{~T}$ and with a long tail of about $10 \mathrm{~T}$. The mean values of hyperfine magnetic field $B_{\text {field }}$ are presented in Table III. The analysis shows that 2 nd and 5 th line disappear within the limits of the error which indicates that the iron magnetic moments are arranged parallel to the external magnetic field. There are no traces of $\alpha-\mathrm{Fe}_{2} \mathrm{O}_{3}$ [20], which is usually very well detected when an external magnetic field is applied.

\section{Conclusion}

The influence of five different surfactants on the magnetic properties of magnetite/maghemite nanoparticles was studied. The TEM images show that the sizes of nanoparticles are in the range from $(14 \pm 2) \mathrm{nm}$ for SA to $(6 \pm 2) \mathrm{nm}$ for AA. The analysis of the X-ray diffraction spectra shows that the nanoparticle size is independent of the kind of surfactant and all equals $(7 \pm 2) \mathrm{nm}$. Such discrepancies are caused by few contributions, which influence the width of the diffraction lines such as: size distribution of prepared nanoparticles, nonstoichiometric, surface effect, distortions of the crystal structure etc. The Mössbauer study at room temperature shows the superparamagnetic doublet in the central part of the spectra and broad magnetic components. The least intensity of the magnetic component was observed in the case of $\mathrm{CA}$ while the highest value was found for SA. The Mössbauer measurements carried out in the external magnetic field of $1.3 \mathrm{~T}$ show how to suppress the superparamagnetic fluctuation and iron magnetic moments are arranged parallel to the applied field.

\section{Acknowledgments}

The work was partially financed by EU funds via a project with contract numbers POPW.01.03.00-20034/09-00, POPW.01.03.00-20-004/11-00, and by NCN found, project number 2014/13/N/ST5/00568.

\section{References}

[1] S.C. McBain, H.H.P. Yiu, J. Dobson, Int. J. Nanomed. 3, 169 (2008).

[2] M. Giersig, M. Hilgendorff, Eur. J. Inorg. Chem. 18, 3571 (2005).

[3] J.B. Haun, T.J. Yoon, H. Lee, H.R. Weissleder, Wiley Interdiscip. Rev. Nanomed. Nanobiotechnol. 2, 291 (2010).

[4] J. Xu, J. Sun, Y. Wang, J. Sheng, F. Wang, M. Sun, Molecules 19, 11465 (2014).

[5] L. Vékás, D. Bica, O. Marinica, Rom. Rep. Phys. 58, 257 (2006).

[6] B. Kalska-Szostko, M. Rogowska, D. Satuła, Coll. Surf. B 111, 656 (2013).

[7] M. Racuciu, D.E. Creanga, A. Airinei, Eur. Phys. J. E 21, 117 (2006).

[8] S. Haracz, M. Hilgendorff, J.D. Rybka, M. Giersig, Nucl. Instrum. Methods Phys. Res. B 364, 120 (2015).

[9] Wahajuddin, S. Arora, Int. J. Nanomed. 7, 3445 (2012).

[10] W. Yu, H. Xie, J. Nanomater. 2012, 1 (2012).

[11] L.L. Vatta, R.D. Sanderson, K.R. Koch, Adv. Mater. 78, 1793 (2006).

[12] K. Petcharoen, A. Sirivat, Mater. Sci. Eng. B SolidState Mater. Adv. Technol. 177, 421 (2012).

[13] B. Kalska-Szostko, M. Cydzik, D. Satuła, M. Giersig, Acta Phys. Pol. A 119, 15 (2011).

[14] B. Kalska-Szostko, U. Wykowska, D. Satula, P. Nordblad, Beilstein J. Nanotechnol. 6, 1385 (2015).

[15] H.El. Ghandoor, H.M. Zidan, M.M.H. Khalil, M.I.M. Ismail, Int. J. Electrochem. Sci. 7, 5734 (2012).

[16] L. Blaney, Leigh Rev. 15, 32 (2007).

[17] U. Klekotka, D. Satuła, S. Spassov, B. KalskaSzostko, Coll. Surf. A Physicochem. Eng. Asp. 537, 452 (2018).

[18] A.K. Zak, W.H.A. Majid, M.E. Abrishami, R. Yousefi, Solid State S 13, 251 (2011).

[19] P.E. Tomaszewski, Phase Transit. 86, 260 (2013).

[20] B. Kalska-Szostko, D. Satuła, W. Olszewski, Curr. Appl. Phys. 15, 226 (2015). 